\title{
Influencia del micrositio y la exposición en la regeneración de bosques de Nothofagus pumilio afectados por Castor canadensis en Tierra del Fuego: un análisis exploratorio
}

\author{
Influence of microsites and exposure in tree regeneration of Nothofagus pumilio \\ forests affected by Castor canadensis in Tierra del Fuego: An exploratory analysis
}

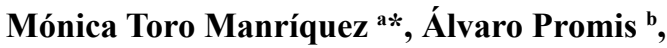 \\ Alejandro Huertas Herrera ${ }^{a}$, Guillermo Martínez Pastur a \\ *Autor de correspondencia: ${ }^{a}$ Laboratorio de Recursos Agroforestales, Centro Austral de Investigaciones Científicas (CADIC), \\ Consejo Nacional de Investigaciones Científicas y Técnicas (CONICET), Houssay 200, Ushuaia (9410), Tierra del Fuego, \\ Argentina. tel.: +54-2901-42-23310, monicatm@cadic-conicet.gob.ar \\ ${ }^{\mathrm{b}}$ Universidad de Chile, Departamento de Silvicultura y Conservación de la Naturaleza, Santiago, Chile.
}

\section{SUMMARY}

Microsites are determinant for seed germination and establishment of forest plants. In Tierra del Fuego, Nothofagus spp. affected by Castor canadensis (beaver) shows a restriction in the regeneration, affecting the natural dynamics of the forest. The objective was to determine the influence of different microsites and exposures, for tree regeneration of $N$. pumilio in an abandoned beaver dam. Two exposures (north-N and south- S) were considered in a forest area that was flooded by beaver action (BI), a forest area affected by cuts (BC) and an area of primary forest (BP) unaffected and contiguous to the previous areas. Ten microsites types were defined in the study area. BP, as a condition without alteration, presented five microsites, of which the highest plant density was litter (H) and bare soil (SD) (60-74 plants $/ \mathrm{m}^{2}$ respectively). BC presented five microsites, with the superior establishment (15.9 plants $\left./ \mathrm{m}^{2}\right)$ on advanced decaying wood (MD-3). Contrary, in BI, the number of plants is lower $\left(4.8\right.$ plants $\left./ \mathrm{m}^{2}\right)$ and it only occurs in edge wood (BT). Plant establishment was higher on S exposure, for all three-forest types due to greater shade. The 100 percent of the plant established in BI (microsite BT) were browsed by Lama guanicoe. It is suggested that the activities of restoration of abandoned beavers use plantations oriented to the most appropriate microsites. Likewise, the exclusion of $L$. guanicoe can accelerate the recovery of affected areas.

Key words: safe site, microsite for regeneration, seedling, primary forest, beaver meadow.

\section{RESUMEN}

Los micrositios son determinantes para la germinación de semillas y el establecimiento de plantas. En Tierra del Fuego, los bosques de Nothofagus spp. afectados por Castor canadensis muestran una restricción en la regeneración, afectando la dinámica natural del bosque. El objetivo fue determinar la influencia de los diferentes micrositios disponibles y la exposición para la regeneración de $N$. pumilio en una castorera abandonada. Se consideraron dos exposiciones (norte $-\mathrm{N}$ y sur $-\mathrm{S}$ ) en un área de bosque que fue inundado por $C$. canadensis (BI), un área de bosque afectado por árboles cortados (BC) y un área de bosque primario (BP) no afectado y contiguo a las áreas anteriores. Se definieron 10 tipos de micrositios en el área de estudio. El BP como condición sin alteración presentó cinco micrositios, de los cuales la mayor densidad de plantas fue en hojarasca (H) y suelo desnudo (SD) $\left(60\right.$ y 74 plantas $/ \mathrm{m}^{2}$, respectivamente). El BC presentó cinco micrositios, con mayor establecimiento (15,9 plantas $\left./ \mathrm{m}^{2}\right)$ sobre madera en descomposición avanzada (MD-3). Contrariamente, en BI la cantidad de plantas es menor (4,8 plantas $\left./ \mathrm{m}^{2}\right)$ y solo se presenta en borde tronco (BT). En la exposición S ocurrió el mayor establecimiento de plantas para los tres tipos de bosque debido al mayor sombreamiento. El 100\% de las plantas establecidas en BI (micrositio BT) fueron ramoneadas por L. guanicoe. Se sugiere que las actividades de restauración de castoreras abandonadas utilicen plantaciones orientadas a los micrositios más adecuados. Asimismo, la exclusión de L. guanicoe aceleraría la recuperación de las áreas afectadas.

Palabras clave: sitio seguro, micrositio para la regeneración, plantas, bosque primario, pradera de castor.

\section{INTRODUCCIÓN}

La restauración de bosques considera varios enfoques, entre los que se cuenta el uso de regeneración natural y la plantación de árboles (Stanturf et al. 2017). En ambos enfoques, la disponibilidad de micrositios es fundamental para el establecimiento de las plantas (Christie y Armesto 2003, Bailey et al. 2012, Valenzuela et al. 2016). Los micrositios para la regeneración, o sitios seguros, son aquellos sectores del suelo del bosque donde las semillas pue- 
den germinar sobre un conjunto de condiciones ambientales adecuadas, asegurando las condiciones necesarias para la germinación de semillas y posterior establecimiento de plantas (Christie y Armesto 2003, Bailey et al. 2012).

En el interior de los bosques se pueden encontrar una gran variedad de micrositios originados por la caída de árboles, troncos y ramas sobre el suelo, el suelo mineral expuesto por disturbio (e.g. zona descalce de árboles caídos), la acumulación de hojarasca y la existencia de flora vascular y no vascular (Christie y Armesto 2003, Kuuluvainen y Kalmari 2003, Bailey et al. 2012). Esta heterogeneidad en el suelo del bosque permite que distintas especies arbóreas logren establecerse según la disponibilidad de micrositios, pudiendo algunas de ellas coexistir en el tiempo (Christie y Armesto 2003). Esta heterogeneidad también se observa en bosques cosechados de Nothofagus pumilio (Poepp. et Endl.) Krasser, donde aquellas áreas cubiertas de restos leñosos, presentan plantas de regeneración con mejor respuesta ecofisiológica y rendimiento respecto a otros microambientes producidos por los tipos de retención de árboles y el grado de impacto de las operaciones de intervención (Martínez Pastur et al. 2014). También se ha documentado para el caso de algunos bosques vírgenes de Nothofagus betuloides (Mirb.) Oerst, que las condiciones microambientales del suelo determinan el reclutamiento de las plantas de regeneración, relacionando a la humedad del suelo con cada microambiente, generando efectos tanto positivos como (suelo desnudo, helecho y musgos creciendo como césped) como efectos negativos (musgos creciendo en forma de esteras, pozos y la cercanía al estrato superior arbóreo) (Martínez Pastur et al. 2012).

Para que el proceso de regeneración de $N$. pumilio sea exitoso, en general, es necesario una cantidad suficiente de semillas viables y condiciones microclimáticas y edáficas adecuadas para la germinación y el desarrollo posterior de las plantas (Martínez Pastur et al. 2007, 2011). Se ha señalado que $N$. pumilio es una especie de tolerancia media, debido a su habilidad de colonización y capacidad de sobrevivir bajo el dosel arbóreo (Martínez Pastur et al. 2007), y que se beneficia de las variaciones ambientales generadas por la cosecha forestal, como los cambios en las propiedades de los suelos, acumulación de restos de madera de gran tamaño y aumento de los niveles de radiación solar (Martínez Pastur et al. 2014).

En este sentido, Caldentey et al. (2009) y Martínez Pastur et al. (2011) han documentado que el crecimiento en altura de plantas de regeneración de $N$. pumilio se vería favorecido con una disminución de la cobertura del dosel arbóreo (entre el 34 al 45\% de cobertura arbórea), lo que permite un mayor ingreso de luz al interior del bosque. Al mismo tiempo, la humedad del suelo también influye fuertemente en el proceso de establecimiento, y su disminución reduce la supervivencia y el crecimiento de las plantas de regeneración (Heinemann et al. 2000, Martínez Pastur et al. 2007, 2011). Por ejemplo, un efecto que se relaciona con la humedad en estos bosques es la acción desecante generada por la exposición al viento, que actúa como un factor limitante en el establecimiento de plantas de regeneración en grandes claros o hacia el límite altitudinal (McIntire et al. 2016). Así, la temperatura, radiación y humedad del suelo son los factores que más afectan al crecimiento y la supervivencia de las plantas en los bosques australes (Heinemman et al. 2000, Martínez Pastur et al. 2007). Un aspecto importante a considerar dentro de la disponibilidad de luz y humedad es cómo influye la exposición sobre estas variables, que a su vez benefician o dificultan la instalación y/o establecimiento de la regeneración bajo distintas coberturas de dosel arbóreo (Heinemman et al. 2000, Martínez Pastur et al. 2011). Además, la dinámica de la regeneración de los bosques de Nothofagus spp. se ajusta a la disminución en la limitación de los recursos como luz y nutrientes del suelo, lo que influye en el aumento del tamaño de las plantas (Promis y Allen 2017).

No obstante, una de las alteraciones que estarían afectando en gran medida la disponibilidad de plantas de regeneración en los bosques de $N$. pumilio de Tierra del Fuego, principalmente en los bosques ribereños (Baldini et al. 2008), es el impacto generado por el castor americano (Castor canadensis Kuhl, 1820). La presencia de C. canadensis ha producido importantes alteraciones ecológicas en estos bosques, tales como la modificación de la morfología e hidrología de cursos de agua y la destrucción del bosque de ribera (Anderson et al. 2009). Esta modificación en estructura puede provocar, además, un aumento en la radiación solar, la temperatura del suelo y la velocidad del viento respecto al bosque original (Promis et al. 2010). También influye sobre la acumulación de sedimentos en áreas inundadas, retención de nutrientes y profundidad de la napa freática (Henn et al. 2014), el cambio de la composición de especies (e.g. rápida colonización de especies intolerantes formando una pradera luego del drenaje) y la dinámica de la regeneración (Martínez Pastur et al. 2006, Henn et al. 2014). Estos cambios que producen la perturbación del $C$. canadensis son similares a las prácticas silvícolas ya que aumentan los niveles radiación solar y luz a nivel del suelo (Promis et al. 2010, Martínez Pastur et al. 2011, Henn et al. 2014).

En bosques de $N$. pumilio el proceso de regeneración está determinado por la existencia de un banco de plantas de regeneración en el sotobosque previo a los impactos o al aporte de semillas nuevas de árboles remanentes o parches de bosques cercanos (Martínez Pastur et al. 2008). La regeneración preinstalada puede responder rápidamente después de la creación de claros de dosel en bosques maduros o por la disminución de cobertura arbórea en bosques manejados (Caldentey et al. 2009, Martínez Pastur et al. 2011). Por lo tanto, al corto plazo se podría esperar que se presenten plantas de regeneración de $N$. pumilio en áreas de bosques ribereños afectados por la acción de C. canadensis y posteriormente abandonados. Sin embargo, a largo plazo ( $>5$ años) se ha observado una tendencia en disminución de densidad de plantas de regeneración en 
praderas de C. canadensis (Martínez Pastur et al. 2006, Henn et al. 2014). El establecimiento y crecimiento de estas plantas, además, se ha visto limitado por el ramoneo efectuado por individuos de Lama guanicoe Müller, hasta en algunos casos las plantas cambian de hábito hacia arbustos deformes (Anderson et al. 2009).

Es así como después del abandono de praderas generadas por C. canadensis, Henn et al. (2014) promueven la restauración activa por medio de plantaciones con N. pumilio, cuya supervivencia sería mayor en zonas donde los árboles fueron cortados, pero no afectado por la inundación, cuyo crecimiento sería similar entre las diferentes zonas de la pradera de $C$. canadensis. Además, para proyectos de reforestación y/o restauración con $N$. pumilio en ambientes con alto estrés, similar a los generados por $C$. canadensis, se estaría recomendando el uso de micrositios de facilitación, tales como arbustos nodrizas y troncos (Valenzuela et al. 2016). En este sentido, Vidal et al. (2015) han realizado plantaciones en micrositios basados en legados biológicos (e.g. estructuras remanentes tales como arbustos, árboles muertos en pie o en el suelo, árboles vivos aislados, o bordes de fragmentos boscosos aislados), que quedan después de la ocurrencia de un disturbio (Franklin 1990), lo que han facilitado la sobrevivencia de las plantas (más del $97 \%$ al año de plantación) mediante la generación de semisombra, la retención de humedad y la protección contra el ramoneo por herbívoros.

Por lo tanto, las distintas estrategias dentro de los planes de restauración deberían considerar la capacidad de $N$. pumilio para regenerar naturalmente y asistir por medio de la reforestación, en caso de ser necesario, tomando en cuenta los cambios generados en el ambiente post-disturbio y los riesgos a los que se debe enfrentar (e.g. protección contra el viento y el ramoneo) (Clewel 2015).

La definición de los micrositios de regeneración forestal puede servir como antecedente para identificar las áreas que podrían recuperarse de forma natural o los lugares donde mejor se podría incorporar una planta por medio de la restauración activa. Por tal motivo, el objetivo de este trabajo fue determinar la influencia de los micrositios de regeneración disponibles para $N$. pumilio en áreas perturbadas y no perturbadas por C. canadensis y a su vez analizar el efecto de dichos micrositios y la exposición sobre la densidad de plantas de regeneración y el efecto del ramoneo por $L$. guanicoe producto de la formación de la pradera. Se toma como caso de estudio (análisis exploratorio) una pradera formada por la acción de $C$. canadensis (área de bosque afectada) y su bosque contiguo (área de bosque sin impacto y medianamente afectado), considerando a su vez, un área de exposición norte con mayor luminosidad, temperatura y menos expuesta a los vientos dominantes, y un área de exposición sur con menor luminosidad y temperatura y más expuesta a los vientos dominantes. Se espera que la exposición influya fuertemente sobre la disponibilidad de plantas de regeneración en los distintos micrositios, diferenciándolos entre las áreas afec- tadas y sin impacto. Estos antecedentes podrían generar nuevos conocimientos para el desarrollo de métodos de regeneración, a partir de una comprensión más profunda de la dinámica de regeneración natural después de las perturbaciones ocasionadas por C. canadensis en los bosques ribereños.

\section{MÉTODOS}

Área de estudio. El estudio se llevó a cabo el año 2012 en el Refugio Vicuña (54ㅇ $08^{\prime}$ 'S, $68^{\circ} 42^{\prime}$ O; 271 m s.n.m.), ubicado en el Parque Natural Karukinka, en la Isla Grande de Tierra del Fuego. El lugar corresponde a una pradera generada por la acción de C. canadensis, que se encontraba abandonada al momento de su estudio, y al bosque contiguo, con sectores afectados por cortes y sin impactos, analizando las exposiciones norte y sur (1,1 ha). Esta zona es similar al ambiente tipo ocho descrito por Briones et al. (2001), en el que por la acción de C. canadensis se modificó la morfología de un arroyo, formándose una laguna, quedando con bosque hacia ambas riberas. $\mathrm{Al}$ momento del muestreo, la pradera tenía seis años luego del abandono de la castorera y la destrucción del dique. El arroyo está encauzado y la laguna ya no existe, presentándose una pradera, en cuya composición predominan especies de la familia Poaceae. Esta área inundada presenta una alta cantidad de restos leñosos dispersos, el dique, árboles caídos y muertos en pie producto de la inundación. El bosque alrededor de la castorera abandonada está compuesto por $N$. pumilio, con una densidad de 373 y 396 árboles/ha, con árboles dominantes de altura entre 18 y $19 \mathrm{~m}$ y área basal entre 85,1 y $91,9 \mathrm{~m}^{2} / \mathrm{ha}$, para las exposiciones $\mathrm{N}$ y S, respectivamente (Toro Manríquez et al. 2014). Entre el bosque y la castorera abandonada, se encuentra un área con restos de árboles cortados, árboles muertos en pie (a causa del anillamiento y descortezamiento generado por $C$. canadensis) y restos de troncos y ramas tendidos sobre el suelo.

Diseño muestreo y evaluación de la regeneración natural por condición de bosque, exposición y micrositio. Para este análisis exploratorio, el área total de estudio (1,8 ha) fue subdividida en: bosque primario (BP), correspondiente a un bosque maduro compuesto por N. pumilio; bosque cortado (BC), que representa el área boscosa donde el C. canadensis realizó corta de árboles, pero no fue inundado por la laguna formada, y bosque inundado (BI) que corresponde al área drenada y convertida en pradera. A su vez estas tres condiciones de bosques fueron subdivididas de acuerdo con su exposición del terreno: exposición norte $(\mathrm{N})$ y exposición sur $(\mathrm{S})$. En cada una de estas áreas (condición de bosque $\times$ exposición) se establecieron cinco parcelas (cuadradas de $900 \mathrm{~m}^{2}$ cada una) en las que se realizaron siete sub-parcelas de $1 \mathrm{~m}^{2}$ de superficie (Caldentey et al. 2009), donde se muestreó la cobertura de suelo, la vegetación herbácea y las plantas de regeneración de 
$N$. pumilio $(5$ parcelas $\times 3$ tipos de bosque $\times 2$ exposiciones). Dentro de estas sub-parcelas se estimó visualmente la superficie ocupada por cada uno de los 10 micrositios disponibles (cuadros 1 y 2) (Kuuluvainen y Kalmari 2003), donde podría establecerse la regeneración. Se identificaron 10 micrositios disponibles (cuadros 1 y 2). Para mejorar la estimación visual de la cobertura de micrositios, la sub-parcela de $1 \mathrm{~m}^{2}$ fue subdivida en 100 cuadrículas de $0,01 \mathrm{~m}^{2}$ cada una. Los troncos que se encontraban sobre el suelo fueron clasificados en tres categorías de acuerdo con su grado de descomposición descritas por Promis et al. (2012), las que consideran la integridad estructural y la consistencia de la madera (cuadro 2).

Además, dentro de las mismas sub-parcelas de $1 \mathrm{~m}^{2} \mathrm{de}$ superficie se calculó el número de plantas de regeneración encontradas. Se consideraron, por separado, las plantas recién germinadas de semilla (del año y con cotiledones a la vista) y las plantas ya establecidas (mayores a 1 año), identificando al mismo tiempo el micrositio sobre el cual cada planta crecía. Posteriormente, se calculó la densidad de plantas por superficie de micrositio y se verificó en cada una de estas la presencia de daño por ramoneo de L. guanicoe. El daño por ramoneo se consideró cuando la planta presentaba cortes en brotes apicales y/o laterales, cambiando en algunos casos su hábito arbóreo al arbustivo. Solamente se clasificó la presencia o ausencia de daño de las plantas en cada micrositio y se calculó el porcentaje de ramoneo $(\%)$.

Análisis estadístico. La cobertura de micrositios (\%) y densidad de plantas por micrositio (plantas $/ \mathrm{m}^{2}$ ) se analizaron por medio de ANDEVA múltiples, considerando como factores principales de análisis la condición de bosque (BP, $\mathrm{BC}, \mathrm{BI})$ y la exposición $(\mathrm{N}, \mathrm{S})$. Las coberturas de micrositio por parcela y la densidad de plantas de regeneración por parcela, se promediaron a partir de las siete sub-parcelas. Esto se realizó para evitar la pseudoreplicación, de manera de diferenciar la parcela de observación (sub-parcela) y la experimental (parcela que contiene las sub-parcelas) (Steel et al. 2013). Asimismo, fue analizada la interacción entre estos factores y se realizaron comparaciones utilizando la prueba de Tukey a posterior, para un nivel de significación del $P<0,05$. Para mejorar la normalidad y reducir la homocedasticidad de las variables (MD-1, MD-2, MD-3 y BT), estas últimas fueron transformadas mediante el $\ln (x+1)$ (Sokal y Rohlf 2000).

Cuadro 1. Descripción de los micrositios estudiados en bosques y praderas generadas por Castor canadensis en los bosques de Nothofagus pumilio.

Description of microsites studied in forests and meadows generated by Castor canadensis in Nothofagus pumilio forests.

\begin{tabular}{|c|c|}
\hline Micrositio (nombre/abreviación) & Definición \\
\hline Hojarasca / H & Hojas que cubren el suelo con presencia de ramas y/o corteza \\
\hline Briófitas / BR & Porción de suelo dominado mayormente por presencia de musgos y/o hepáticas \\
\hline Helechos / HL & Porción de suelo dominado mayormente por Blechnum penna-marina (Poir.) Kuhn \\
\hline Herbáceas / HB & Área cubierta mayormente por especies de monocotiledóneas y dicotiledóneas con hábito herbáceo \\
\hline Suelo desnudo / SD & Suelo mineral expuesto, incluyendo revolcaderos de Lama guanicoe \\
\hline $\begin{array}{l}\text { Borde troncos con briófitas y } \\
\text { herbáceas o suelo desnudo / BT }\end{array}$ & Sectores con protección de troncos caídos con distintos grados y/o tipos de cubierta \\
\hline Otros / O & Pozo de desarraigo, montículo de desarraigo, suelo cubierto de arbustos, heces de L. guanicoe \\
\hline
\end{tabular}

Cuadro 2. Etapas de descomposición de los troncos caídos basado en Promis et al. (2012).

Stages of decomposition of fallen trunks based on Promis et al. (2012).

Micrositio (nombre/abreviación)

Madera en descomposición grado 1 / MD-1

Madera en descomposición grado 2 / MD-2

Madera en descomposición grado 3 / MD-3
Definición e identificación

Tronco caído con madera dura. Un cuchillo solo puede penetrar menos de $1 \mathrm{~cm}$

Tronco caído con madera medianamente dura a suave. Un cuchillo puede penetrar menos de $2 \mathrm{~cm}$

Tronco caído con madera suave a muy suave. Un cuchillo puede penetrar más de $2 \mathrm{~cm}$ 


\section{RESULTADOS}

Micrositios adecuados para la regeneración. Existe una variedad de micrositios que es posible encontrar en cada uno de los ambientes, cuya composición varía de acuerdo con sus características y que pueden presentarse como posibles micrositios adecuados para la regeneración (cuadros 1 y 2). Sin embargo, dentro del muestreo, no se identificaron todos los micrositios posibles de encontrar en estos ambientes, e.g. en bosque inundado se encontraron nueve de los 10 micrositios y en el interior del bosque primario se encontraron siete de los 10 micrositios. El micrositio más abundante fue de hojarasca, tanto por condiciones de tipo de bosques y por exposiciones (cuadro 3).

Se encontraron diferencias significativas entre las coberturas de algunos micrositios presentes por tipo de bosque y exposición. En el interior del bosque primario, los micrositios que presentaron una mayor cobertura estadísticamente respecto las otras condiciones de sitio son hojarasca, suelo desnudo, helechos y otros (cuadro 3). En la zona del bosque inundado, la cobertura de herbáceas y presencias de borde de troncos fueron mayores estadísticamente, mientras que en la zona del bosque cortado la cobertura de micrositios se presentó casi siempre con valores intermedios entre bosque primario y bosque inundado. La madera en descomposición grado 1 fue estadísticamente mayor en el bosque inundado y bosque cortado, respecto al bosque primario y madera en descomposición grado 3 en bosque primario y bosque cortado respecto al bosque inundado (cuadro 3). En la zona expuesta hacia el sur, los micrositios hojarasca, suelo desnudo y herbáceas, tuvieron mayor cobertura estadísticamente respecto a la exposición norte. En la exposición norte, la cobertura de borde de troncos y otros, fueron mayores respecto a la exposición sur (cuadro 3).

Regeneración natural en cada micrositio. La mayor cantidad de plantas de $N$. pumilio establecidas se encuentra en el interior del bosque primario (cuadro 4). En solo seis de los micrositios se han registrado establecimiento de plantas (cuadro 4 y figura 1), de las cuales existen diferencias significativas entre tipos de bosque y exposición. Los micrositios hojarasca, briófitas, suelo desnudo y madera en descomposición grado 3 fueron donde hay significativamente mayor establecimiento de plantas en el bosque primario, respecto de bosque cortado y bosque inundado (cuadro 4). El suelo desnudo y hojarasca fueron los micrositios que presentaron mayor densidad de plantas regeneradas en bosque primario, mientras que bosque cortado favoreció en establecimiento de plantas principalmente en

Cuadro 3. ANDEVA múltiple para la cobertura de micrositios (\%) presentes en el área estudiada de acuerdo con el tipo de bosque (BP: bosque primario; BC: bosque cortado por Castor canadensis; BI: bosque afectado por inundación) y a la exposición (N: norte; S: sur).

Multiple ANOVA for the coverage of microsites (\%) present in the study area according to forest type (BP: primary forest, BC: forest cut by Castor canadensis, BI: forest affected by flood) and exposure (N: north, S: south).

\begin{tabular}{|c|c|c|c|c|c|c|c|c|c|c|c|}
\hline Factor & $\begin{array}{l}\text { Fuente de } \\
\text { variación }\end{array}$ & $\mathrm{H}$ & BR & SD & $\mathrm{HL}$ & HB & MD-1 & MD-2 & MD-3 & BT & $\mathrm{O}$ \\
\hline \multirow{5}{*}{$\begin{array}{l}\text { A: Tipo de } \\
\text { bosque }\end{array}$} & $\mathrm{BP}$ & $17,12 \mathrm{c}$ & $4,94 \mathrm{a}$ & $4,02 \mathrm{~b}$ & $6,94 \mathrm{~b}$ & $28,51 \mathrm{a}$ & $0,00 \mathrm{a}$ & 0,00 & $11,00 \mathrm{~b}$ & $0,00 \mathrm{a}$ & $27,47 \mathrm{~b}$ \\
\hline & $\mathrm{BC}$ & $7,58 \mathrm{~b}$ & $18,21 \mathrm{~b}$ & $1,40 \mathrm{ab}$ & $2,66 \mathrm{ab}$ & $35,76 \mathrm{a}$ & $8,60 \mathrm{~b}$ & 1,23 & $9,78 \mathrm{~b}$ & $3,59 \mathrm{ab}$ & $11,19 \mathrm{a}$ \\
\hline & BI & $1,30 \mathrm{a}$ & $10,45 \mathrm{ab}$ & $0,68 \mathrm{a}$ & $0,76 \mathrm{a}$ & $55,28 \mathrm{~b}$ & $13,45 \mathrm{~b}$ & 1,56 & $0,00 \mathrm{a}$ & $9,34 \mathrm{~b}$ & $7,18 \mathrm{a}$ \\
\hline & $\mathrm{F}$ & 21,96 & 4,60 & 4,78 & 4,93 & 14,74 & 10,66 & 1,39 & 13,47 & 3,50 & 10,41 \\
\hline & $P$ & $<0,001 * * *$ & $0,020^{*}$ & $0,018^{*}$ & $0,016^{*}$ & $<0,001 * * *$ & $<0,001 * * *$ & $=0,268 \mathrm{~ns}$ & $<0,001^{*}$ & $0,046^{*}$ & $<0,001^{*}$ \\
\hline \multirow{4}{*}{ B: Exposición } & $\mathrm{N}$ & $5,76 \mathrm{a}$ & 10,33 & $0,87 \mathrm{a}$ & 2,69 & 34,35 a & 6,31 & 0,61 & 7,09 & $7,67 \mathrm{~b}$ & $24,32 \mathrm{~b}$ \\
\hline & $\mathrm{S}$ & $11,58 \mathrm{~b}$ & 12,06 & $3,20 \mathrm{~b}$ & 4,21 & $45,15 \mathrm{~b}$ & 8,39 & 1,25 & 6,76 & $0,95 \mathrm{a}$ & $6,45 \mathrm{a}$ \\
\hline & $\mathrm{F}$ & 8,80 & 0,23 & 6,27 & 0,85 & 6,68 & 0,74 & 0,62 & 0,03 & 5,33 & 16,94 \\
\hline & $P$ & $0,007 * *$ & $0,635 \mathrm{~ns}$ & $0,027^{*}$ & $0,366 \mathrm{~ns}$ & $0,016^{* *}$ & $0,398 \mathrm{~ns}$ & $0,437 \mathrm{~ns}$ & $0,861 \mathrm{~ns}$ & $0,030^{*}$ & $<0,001 * * *$ \\
\hline \multirow{2}{*}{$\mathrm{A} \times \mathrm{B}$} & $\mathrm{F}$ & 2,57 & 1,58 & 3,11 & 0,02 & 2,90 & 0,78 & 1,33 & 3,63 & 2,25 & 10,97 \\
\hline & $P$ & $0,097 \mathrm{~ns}$ & $0,226 \mathrm{~ns}$ & $0,063 \mathrm{~ns}$ & $0,985 \mathrm{~ns}$ & $0,075 \mathrm{~ns}$ & $0,471 \mathrm{~ns}$ & $0,285 \mathrm{~ns}$ & $0,042^{*}$ & $0,127 \mathrm{~ns}$ & $<0,001 * * *$ \\
\hline
\end{tabular}

F: Prueba de Fisher; $P$ : probabilidades límites en ANDEVA múltiple. *: $P<0,05 ; * *: P<0,01 ; * * *: P<0,001 ;$ ns $=P>0,05$. Letras diferentes en cada columna indican diferencias significativas (Tukey, $P<0,05$ ). H: Hojarasca; BR: Briófitas; SD: Suelo desnudo; HL: Helechos; HB: Herbáceas; MD-1: Madera en descomposición grado 1; MD-2: Madera en descomposición grado 2; MD-3: Madera en descomposición grado 3; BT: borde tronco; O: otros.

F: Fisher's test; $P$ : limit probabilities in multiple ANOVA. *: $P<0.05 ; * *: P<0.01 ; * *: P<0.001 ; \mathrm{ns}=P>0.05$. Different letters in each column indicate significant differences (Tukey, $P<0.05$ ). H: Leaf litter; BR: Bryophytes; SD: Bare soil; HL: Ferns; HB: Herbaceous; MD-1: Decaying wood grade; MD-2: Decaying wood grade 2; MD-3: Decaying wood grade 3 wood; BT: trunk edge; O: others. 
madera en descomposición grado 3. El micrositio borde de troncos fue utilizado para el establecimiento de plantas en el bosque inundado y en el bosque cortado (en el bosque primario no se presentó como micrositio) (cuadro 4). Respecto de las exposiciones, las mayores densidades de plantas de regeneración se establecieron hacia la exposición sur, donde casi todos los micrositios presentaron mayor número de plantas establecidas respecto a la exposición norte (cuadro 4 y figura 1 ).

Efecto del ramoneo de L. guanicoe sobre la regeneración en distintos micrositios. El ramoneo por L. guanicoe presentó diferencias significativas en todos los tipos de bosques, pero estas diferencias no se observaron en las exposiciones (cuadro 5). Los daños se observaron en cuatro micrositios (hojarasca, madera en descomposición grado 3 , herbáceas y borde de troncos), donde fue mayor en hojarasca y madera en descomposición grado 3 en el bosque primario respecto a los otros tipos de bosques (cuadro 5). En el micrositio herbáceo, el ramoneo fue estadísticamen- te mayor en el bosque cortado respecto a los otros tipos de bosques. Mientras que en el micrositio borde de troncos, el mayor porcentaje de ramoneo fue en el bosque inundado respecto a los otros tipos de bosques (cuadro 5).

\section{DISCUSIÓN}

Influencia de los micrositios de regeneración disponibles para N. pumilio en áreas no perturbadas por C. canadensis. Algunos estudios han mostrado la importancia del ciclo de regeneración de $N$. pumilio en la dinámica natural del bosque. Martínez Pastur et al. (2008) y Toro Manríquez et al. (2016) demuestran que para esta especie se presentan años de alta o baja semillazón, con variabilidad en la calidad de las semillas bajo distintas condiciones y localizaciones del paisaje, que puede mostrar un potencial muy variable de instalación de plantas. El que se establezca un banco de plantas en el sotobosque depende de la estructura del dosel arbóreo, las características del suelo, del sotobosque y de variables de contexto espacial, como la ex-
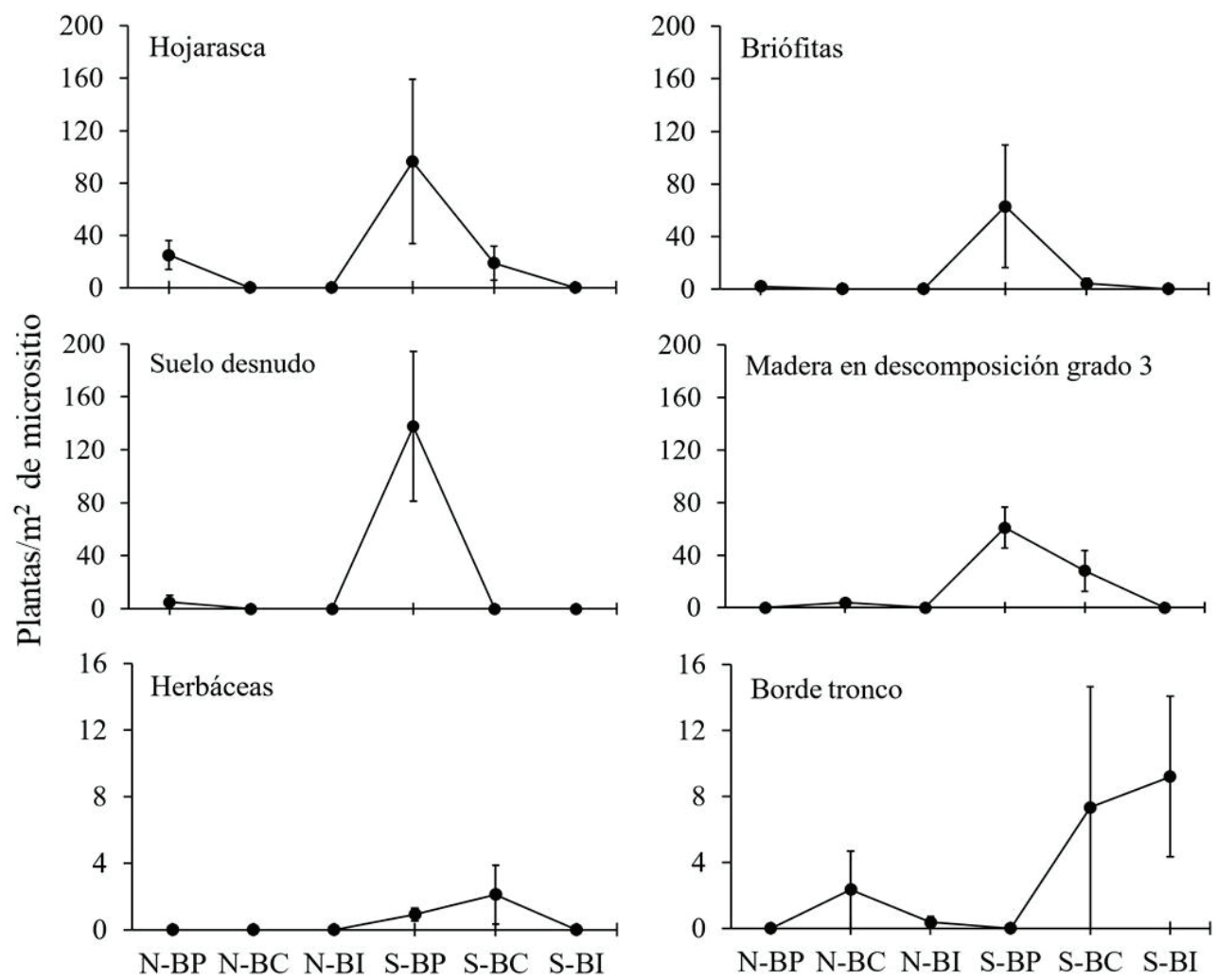

Exposición-Tipo de Bosque

Figura 1.Densidad de plantas de N. pumilio $\left(\operatorname{plantas} / \mathrm{m}^{2}\right.$ ) para micrositios hojarasca, briófitas, suelo desnudo, herbáceas, madera en descomposición grado 3 y borde tronco, distribuidos por la combinación de exposiciones (N: norte; S: sur) y tipo de bosque (BP: bosque primario; BC: bosque cortado por acción de Castor canadensis; BI: bosque inundado). Barras muestran el error estándar.

Density of $N$. pumilio plants (plants $/ \mathrm{m}^{2}$ ) for microsites litter, bryophytes, bare soil, herbaceous, decaying wood grade 3, wood edge, distributed by the combination of exposures (N: north, S: south) and forest type (BP: primary forest, BC: forest cut by Castor canadensis, BI: flooded forest). Bars show the standard error. 
Cuadro 4. ANDEVA múltiple para la densidad media de plantas de $N$. pumilio (plantas $/ \mathrm{m}^{2}$ ) de micrositio por tipo de bosque (BP: bosque primario; BC: bosque cortado por acción Castor canadensis; BI: bosque inundado) y exposición (N: norte; S: sur).

Multiple ANOVA for the average plant density of Nothofagus pumilio (plants/ $\mathrm{m}^{2}$ ) of microsite by forest type (BP: primary forest, $\mathrm{BC}$ : forest cut by action Castor canadensis, BI: flooded forest) and exposure (N: north; S: south).

\begin{tabular}{|c|c|c|c|c|c|c|c|}
\hline Factor & Fuente de variación & $\mathrm{H}$ & $\mathrm{BR}$ & SD & HB & MD-3 & BT \\
\hline \multirow{5}{*}{ A: Tipo de bosque } & $\mathrm{BP}$ & $60,6 \mathrm{~b}$ & $32,4 \mathrm{~b}$ & $74,4 \mathrm{~b}$ & 0,5 & $30,4 \mathrm{~b}$ & 0,0 \\
\hline & $\mathrm{BC}$ & $9,4 \mathrm{a}$ & $2,0 \mathrm{a}$ & $0,0 \mathrm{a}$ & 1,1 & $15,9 \mathrm{~b}$ & 4,8 \\
\hline & $\mathrm{BI}$ & $0,0 \mathrm{a}$ & $0,0 \mathrm{a}$ & $0,0 \mathrm{a}$ & 0,0 & $0,0 \mathrm{a}$ & 4,8 \\
\hline & $\mathrm{F}$ & 16,37 & 1,66 & 50,96 & 1,42 & 14,37 & 1,08 \\
\hline & $P$ & $<0,001 * * *$ & $0,021 *$ & $<0,001 * * *$ & $0,262 \mathrm{~ns}$ & $<0,001 * * *$ & $0,350 \mathrm{~ns}$ \\
\hline \multirow{4}{*}{ B: Exposición } & $\mathrm{N}$ & $8,3 \mathrm{a}$ & $0,7 \mathrm{a}$ & $1,7 \mathrm{a}$ & $0,0 \mathrm{a}$ & $1,28 \mathrm{a}$ & 0,9 \\
\hline & $\mathrm{S}$ & $37,4 \mathrm{~b}$ & $22,3 b$ & $45,9 \mathrm{~b}$ & $1,1 \mathrm{~b}$ & $29,6 \mathrm{~b}$ & 5,5 \\
\hline & $\mathrm{F}$ & 5,57 & 1,73 & 28,9 & 5,58 & 31,73 & 2,35 \\
\hline & $P$ & $0,027^{*}$ & $0,028^{*}$ & $<0,001 * * *$ & $0,026^{*}$ & $<0,001 * * *$ & $0,137 \mathrm{~ns}$ \\
\hline \multirow{2}{*}{$\mathrm{A} \times \mathrm{B}$} & $\mathrm{F}$ & 1,42 & 1,569 & 28,9 & 1,42 & 12,15 & 0,71 \\
\hline & $P$ & $0,262 \mathrm{~ns}$ & $0,228 \mathrm{~ns}$ & $<0,001 * * *$ & $0,262 \mathrm{~ns}$ & $<0,001 * * *$ & $0,501 \mathrm{~ns}$ \\
\hline
\end{tabular}

F: Prueba de Fisher; $P$ : probabilidades límites en ANDEVA múltiple. *: $P<0,05 ; * *: P<0,01$; **: $P<0,001$; ns $=P>0,05$. Letras diferentes en cada columna indican diferencias significativas (Tukey, $P<0,05$ ). H: hojarasca; BR: briófitas; SD: suelo desnudo; HB: herbáceas; MD-3: madera en descomposición grado 3; BT: borde tronco.

F: Fisher's test; $P$ : limit probabilities in multiple ANOVA. *: $P<0.05 ; * *: P<0.01 ; * *: P<0.001 ; \mathrm{ns}=P>0.05$. Different letters in each column indicate significant differences (Tukey, $P<0.05$ ). H: Leaf litter; BR: Bryophytes; SD: Bare soil; HB: Herbaceous; MD-3: Decaying wood grade 3; BT: wood edge.

Cuadro 5. ANDEVA múltiple para la proporción de plantas de regeneración de Nothofagus pumilio ramoneadas por Lama guanicoe (\%) establecidas en diferentes micrositios por tipo de bosque (BP: bosque primario; BC: bosque cortado por acción Castor canadensis; BI: bosque inundado) y exposición (N: norte; $\mathrm{S}$ : sur).

Multiple ANOVA for the proportion of regeneration plants of Nothofagus pumilio browsed by Lama guanicoe (\%) established in different microsites by forest type (BP: primary forest, BC: forest cut by action Castor canadensis, BI: flooded forest) and exposure (N: north; S: south).

\begin{tabular}{|c|c|c|c|c|c|}
\hline Factor & Fuente de variación & $\mathrm{H}$ & MD-3 & $\mathrm{HB}$ & BT \\
\hline \multirow{5}{*}{ A: Tipo de bosque } & $\mathrm{BP}$ & $23,4 \mathrm{~b}$ & $32,9 \mathrm{~b}$ & $0,0 \mathrm{a}$ & $0 \mathrm{a}$ \\
\hline & $\mathrm{BC}$ & $18,1 \mathrm{ab}$ & $20,0 \mathrm{ab}$ & $61,8 \mathrm{~b}$ & $15 \mathrm{a}$ \\
\hline & $\mathrm{BI}$ & $0,0 \mathrm{a}$ & $0,0 \mathrm{a}$ & $0,0 \mathrm{a}$ & $100 \mathrm{~b}$ \\
\hline & $\mathrm{F}$ & 5,58 & 7,60 & 20,56 & 15,22 \\
\hline & $P$ & $0,010 * *$ & $0,003 * *$ & $<0,001 * * *$ & $<0,001 * * *$ \\
\hline \multirow{4}{*}{ B: Exposición } & $\mathrm{N}$ & 25,8 & 22,5 & 26,7 & 33 \\
\hline & $\mathrm{S}$ & 56,3 & 12,8 & 14,5 & 30 \\
\hline & $\mathrm{F}$ & 3,36 & 0,90 & 1,00 & 0,02 \\
\hline & $P$ & $0,109 \mathrm{~ns}$ & $0,352 \mathrm{~ns}$ & $0,328 \mathrm{~ns}$ & $0,877 \mathrm{~ns}$ \\
\hline \multirow{2}{*}{$A \times B$} & $\mathrm{~F}$ & 1,61 & 2,12 & 1,00 & 0,02 \\
\hline & $P$ & $0,221 \mathrm{~ns}$ & $0,142 \mathrm{~ns}$ & $0,384 \mathrm{~ns}$ & $0,976 \mathrm{~ns}$ \\
\hline
\end{tabular}

F: Prueba de Fisher; $P$ : probabilidades límites en ANDEVA múltiple. *: $P<0,05 ; * *: P<0,01 ; * * *: P<0,001 ;$ ns $=P>0,05$. Letras diferentes en cada columna indican diferencias significativas (Tukey, $P<0,05$ ). H: hojarasca; MD-3: madera en descomposición grado 3; HB: herbáceas BT: borde tronco. F: Fisher's test; $P$ : limit probabilities in multiple ANOVA. *: $P<0.05 ; * *: P<0.01 ; * *: P<0.001 ; \mathrm{ns}=P>0.05$. Different letters in each column indicate significant differences (Tukey, $P<0.05$ ). H: Leaf litter; BR: Bryophytes; MD-3: Decaying wood grade 3; HB: Herbaceous; BT: wood edge. 
posición, pendiente y altitud, propiedades del suelo y a la radiación solar (Caldentey et al. 2009, Promis et al. 2010, Martínez Pastur et al. 2011). El área no perturbada que corresponde al bosque primario, presenta la menor cantidad de micrositios, pero concentra una mayor densidad de plantas establecidas. La mayor cantidad de micrositios presentes en el bosque primario fueron: hojarasca, briófitas, suelo desnudo y madera en descomposición grado 3. Naturalmente en el bosque, las plantas de regeneración de $N$. pumilio son capaces de germinar y establecerse solamente cuando hay una buena disponibilidad de hojarasca, madera en descomposición y suelo mineral o desnudo (Christie y Armesto 2003). Sin embargo, los resultados de este estudio muestran que los micrositios que originalmente se encuentran presentes en el bosque primario, disminuyen gradualmente en el bosque cortado y cambian totalmente en el bosque inundado.

Influencia de los micrositios de regeneración disponibles para $N$. pumilio en áreas perturbadas por $C$. canadensis.Los resultados de este estudio indican que en los tres tipos de bosques asociados a un disturbio por acción del C. canadensis, la oferta de micrositios en el bosque inundado es mayor que en bosque cortado y bosque primario. Sin embargo, esta mayor oferta no implica que estos estén disponibles para la regeneración arbórea en el bosque inundado. Por el contrario, el bosque primario presenta la menor cantidad de micrositios, pero concentra una mayor densidad de plantas establecidas. Una posible explicación para esto podría ser que el fuerte impacto sobre la estructura del bosque y el suelo, hace que en el área los micrositios no se presenten de igual proporción que dentro del bosque original, y, por ende, las condiciones para el desarrollo y la sucesión del bosque sean completamente diferentes. Otra posible explicación para esto es que no existe una cobertura de dosel, necesaria para la regeneración después de un impacto (Caldentey et al. 2009, Martínez Pastur et al. 2011).

Un hallazgo interesante es que los micrositios presentes en exposición sur presentaron mayor establecimiento de plantas de regeneración. De acuerdo con Heinemann et al. (2000) y Martínez Pastur et al. (2011), este resultado puede explicarse por el hecho de que factores que condicionan a las plantas, como sombreamiento y humedad, son muy favorables para la regeneración de N. pumilio. En praderas formadas por acción de C. canadensis o en las prácticas silviculturales, los principales cambios generados por la disminución de la cobertura de dosel son el aumento de la luz incidente y humedad que alcanza el nivel del suelo, que implica a efectos directos sobre la dinámica natural de los bosques de N. pumilio (Martínez Pastur et al. 2011). Respecto a ello, estudios han demostrado que, después de que la castorera es abandonada y drenada, factores como la acumulación de sedimentos y el cambio del nivel freático en el suelo incrementan la disponibilidad de recursos favorables para la colonización de plantas herbáceas, principalmente monocotiledóneas (muchas de ellas exóticas), siendo estos un conductor de cambio para que prospere una pradera y no un bosque (Baldini et al. 2008, Martínez Pastur et al. 2006).

En el bosque inundado, el micrositio más favorable fue el de borde de troncos. La presencia de troncos incrementa la disponibilidad de micrositios óptimos para regeneración del bosque, pues los bordes de los troncos son favorables para el establecimiento de plantas. Así, aunque a una baja densidad, estos legados biológicos del bosque desempeñan un papel fundamental en la dinámica del ecosistema (e.g. sombreamiento y proporción de humedad necesaria). El uso de micrositios asociado a restos leñosos también se ha dado en el área del bosque cortado, donde la madera en avanzada descomposición alberga la mayor densidad de plantas de regeneración. Numerosos estudios en bosques con y sin disturbio han encontrado que los micrositios asociados a madera actúan como sitios seguros (safe sites) o nodrizas (Kuuluvainen y Kalmari 2003, Bailey et al. 2012), esto debido a las condiciones microclimáticas (humedad y luz) que proveen, ya sea por la descomposición de la madera o su cercanía a los bordes de esta; situación que se presenta en las áreas bajo impacto en exposición sur en este estudio. Además, según Kuuluvainen y Kalmari (2003), la asociación a la madera disminuye la competencia con otras plantas, lo cual es un factor importante a considerar para la regeneración del bosque en estos ambientes susceptibles a la invasión de praderas. En este contexto, Vidal et al. (2015) y Valenzuela et al. (2016) sugieren que el uso de arbustos o restos de troncos mejoran la supervivencia de plantas, tanto en la regeneración natural del bosque como en la restauración ecológica. Por consiguiente, este tipo de micrositios son considerados estructuras que actúan como facilitadores en el establecimiento inicial de las plantas de Nothofagus spp. (Valenzuela et al. 2016).

Limitaciones a las plantas de regeneración por ramoneo de L. guanicoe. Según Henn et al. (2014), en castoreras abandonadas, la cobertura plantas de herbáceas es un agente de competencia contra la regeneración arbórea, sumado a que, en una primera instancia, esta cobertura hace que se pierda la capacidad del suelo como cama de semillas. Se ha reportado que estos espacios se transforman en una pradera de forrajeo que favorece a $L$. guanicoe (Baldini et al. 2008), y. en concordancia, nuestros resultados indican que la totalidad de las plantas de regeneración establecidas en el bosque inundado se encuentran ramoneadas. Se ha estudiado que no existe una relación positiva entre la apertura del bosque y la intensidad del ramoneo por L. guanicoe (Cavieres y Fajardo 2005). Pese a ello, la totalidad de plantas ramoneadas en el área inundada puede estar relacionada a un mayor movimiento y densidad de L. guanicoe, lo que ejercerían esta mayor presión sobre el establecimiento de plantas dentro de esta área. Por lo que, en la medida que las plantas de regeneración de $N$. pumilio sobresalen desde la cobertura de las plantas herbácea, los efectos del L. guanicoe sobre el ramoneo de las plantas deberían ser considerados dentro de los planes de restauración (Henn et al. 2014). 
CONCLUSIONES Y CONSIDERACIONES PARA LA RESTAURACIÓN DE PRADERA DE C. CANADENSIS

Los resultados de este estudio pueden ayudar a entender la oferta de micrositios en los sectores de bosque con impacto y $\sin$ impacto de $C$. canadensis, aspectos que deberían ser explorados en otras castoreras, para obtener patrones generales. Este impacto en bosque inundado ha generado la influencia de la regeneración establecida en un nuevo micrositio que no se presenta en el bosque primario, como es el asociado a bordes de tronco, ayudando a comprender como responde la dinámica natural de la regeneración frente a estas condiciones. Es interesante considerar como la exposición sur presenta mejores condiciones de micrositios en las áreas perturbadas. Es importante destacar el efecto de L. guanicoe, ya que este afecta el crecimiento de las plantas. Se recomienda para futuras investigaciones abordar este tema, principalmente en las zonas más próximas a los rodales donde existen los micrositios de mayor establecimiento de plantas de regeneración de $N$. pumilio, considerando las características microclimáticas generadas por la exposición. Este estudio presenta información esencial para iniciar planes de restauración pasiva (e.g. manejo y protección de la regeneración natural ya establecida) y activa (e.g. incorporación de plantas) a partir de los micrositios de regeneración natural encontrados en sectores afectados por C. canadensis, tal como los bordes de troncos caídos. Estos aspectos podrían favorecer las acciones de reforestación en bosques de $N$. pumilio. Se debe excluir la presencia de individuos de L. guanicoe, para evitar mortalidad, cambio de forma y/o crecimiento de las plantas de regeneración. Pese a la limitante que se puede generar al estudiar una condición de castorera, este estudio puede servir de aspecto metodológico para evaluar a nivel general aquellos micrositios de establecimiento de plantas de regeneración en áreas bajo distintos agentes de disturbios (e.g. viento, incendios). Es necesario ampliar este tipo estudios a otras condiciones de castoreras, las cuales pueden presentar otros tipos de micrositios y legados biológicos que deberían ser evaluados y considerados.

\section{AGRADECIMIENTOS}

Los autores agradecemos a Wildlife Conservation Society (Parque Natural Karukinka) y personal de Vicuña, al Grupo de Investigación Aplicada en Ecología y Conservación de la Vegetación (ECOVEG) de la Universidad de Chile y al Centro Austral de Investigaciones Científicas (CADIC, CONICET) por su apoyo durante la realización de este trabajo. Al honorable cónsul de Chile en Ushuaia, Tierra del Fuego-Argentina, Sr. Luciano Parodi por sus incentivos para la colaboración Binacional entre Chile-Argentina.

\section{REFERENCIAS}

Anderson CB, G Martínez Pastur, MV Lencinas, PA Wallem, MC Moorman, AD Rosemond. 2009. Do introduced North American beavers Castor canadensis engineer differently in southern South America? An overview with implications for restoration. Mammal Review 39(1):33-52. doi.org/10.1111/j.1365-2907.2008.00136.x.

Bailey T, N Davidson, D Close. 2012. Understanding the regeneration niche: Microsite attributes and recruitment of eucalypts in dry forests. Forest Ecology and Management 269: 229-238. doi.org/10.1016/j.foreco.2011.12.021.

Baldini A, J Oltremari, M Ramírez. 2008. Impacto del castor (Castor canadensis, Rodentia) en bosques de $N$. pumilio (Nothofagus pumilio) de Tierra del Fuego, Chile. Bosque 29(2): 162-169. doi.org/10.4067/S0717-92002008000200009.

Briones M, R Schlatter, A Wolodarsky, C Venegas. 2001. Clasificación ambiental para hábitat de Castor canadensis (Kuhl 1820, Rodentia), de acuerdo a características de cuencas en uno de Tierra del Fuego. Anales del Instituto de la Patagonia 29: 75-93.

Caldentey J, H Mayer, M Ibarra, A Promis. 2009. The effects of a regeneration felling on photosynthetic photon flux density and regeneration growth in a Nothofagus pumilio forest. European Journal of Forest Research 128(1): 75-84. doi. org/10.1007/s10342-008-0240-8.

Cavieres LA, A Fajardo. 2005. Browsing by guanaco (Lama guanicoe) on Nothofagus pumilio forest gaps in Tierra del Fuego, Chile. Forest Ecology and Management 204:237-248. doi.org/10.1016/j.foreco.2004.09.004.

Clewel AF. 2015. Ecological restoration principles relative to Nothofagus pumilio (Poepp. \& Endl.) Krasser (Nothofagaceae) forest restoration. Anales del Instituto de la Patagonia 43(1): 123-126. doi.org/10.4067/S0718686X2015000100010.

Christie D, J Armesto. 2003. Regeneration microsites and tree species coexistence in temperate rain forest of Chiloé Island, Chile. Journal of Ecology 91(5): 776-784. doi. org/10.1046/j.1365-2745.2003.00813.x.

Franklin JF. 1990. Biological legacies: A critical management concept from Mount St. Helens, Transitions of the 55th North American Wildlife and Natural Resource Conference: 216-219.

Heinemann K, T Kitzberger, T Veblen. 2000. Influences of gap microheterogeneity on the regeneration of Nothofagus pumilio in a xeric old-growth forest of north-western Patagonia, Argentina. Canadian Journal of Forest Research 30(1): 25-31. doi.org/10.1139/x99-181.

Henn JJ, CB Anderson, G Kreps, MV Lencinas, R Soler, G Martínez Pastur. 2014. Determining abiotic and biotic drivers that limit active riparian forest restoration in abandoned beaver meadows in Tierra del Fuego. Ecological Restoration 32(4): 369-378. doi.org/10.3368/er.32.4.369.

Kuuluvainen T, R Kalmari. 2003. Regeneration microsites of Picea abies seedlings in a windthrow area of a boreal oldgrowth forest in southern Finland. Annales Botanici Fennici 40: 401-413.

Martínez Pastur G, M Lencinas, J Escobar, P Quiroga, L Malmierca, M Lizarralde. 2006. Understorey succession in Nothofagus forests in Tierra del Fuego (Argentina) affected by Castor canadensis. Journal of Applied Vegetation Science 9(1): 143-154. doi.org/10.1658/1402-2001(2006)9[143:US INFI]2.0.CO;2.

Martínez Pastur G, MV Lencinas, P Peri, M Arena. 2007. Photosynthetic plasticity of Nothofagus pumilio seedlings 
to light intensity and soil moisture. Forest Ecology and Management 243(2): 274-282. doi.org/10.1016/j.foreco.2007.03.034.

Martínez Pastur G, MV Lencinas, PL Peri, JM Cellini. 2008. Flowering and seeding patterns in unmanaged and managed Nothofagus pumilio forests with a silvicultural variable retention system. Forstarchiv 79: 60-65.

Martínez Pastur G, JM Cellini, MV Lencinas, M Barrera, PL Peri. 2011. Environmental variables influencing regeneration of Nothofagus pumilio in a system with combined aggregated and dispersed retention. Forest Ecology and Management 261(1): 78-86. doi.org/10.1016/j.foreco.2010.10.002.

Martínez Pastur G, C Jordán, MV Lencinas, R Soler, H Ivancich, G Kreps. 2012. Landscape and microenvironmental conditions influence over regeneration dynamics in old-growth Nothofagus betuloides Southern Patagonian forests. Plant Biosyst 146(1): 201-213. doi.org/10.1080/11263504.2011.650725.

Martínez Pastur G, R Soler, JM Cellini, MV Lencinas, P Peri, MG Nayland. 2014. Survival and growth of Nothofagus pumilio seedlings under several microenvironments after variable retention harvesting in southern Patagonian forests. Annals of Forest Science 71(3): 349-362. doi.org/10.1007/ s13595-013-0343-3.

McIntire EJB, FI Piper, A Fajardo. 2016. Wind exposure and light exposure, more than elevation-related temperature, limit tree line seedling abundance on three continents. Journal of Ecology 104(5): 1379-1390. doi.org/10.1111/13652745.12599.

Promis A, J Caldentey, M Ibarra. 2010. Microclima en el interior de un bosque de Nothofagus pumilio y el efecto de una corta de regeneración. Bosque 31(2): 129-139. doi.org/10.4067/ S0717-92002010000200006.

Promis A, S Gartner, A Reif, G Cruz. 2012. Effects of canopy gaps on forest floor vascular and non-vascular plant species composition and diversity in an uneven-aged Nothofagus betuloides forest in Tierra del Fuego, Chile. Community Ecology 13(2): 145-154. doi.org/ 10.1556/ ComEc.13.2012.2.3

Promis A, RB Allen. 2017. Tree seedlings respond to both light and soil nutrients in a Patagonian evergreen-deciduous forest. PLoS ONE 12(11): e0188686. doi.org/10.1371/journal.pone. 0188686 .

Sokal RR, FJ Rohlf. 2000. Biometry. The principles and practice of statistics in biological research. Third Edition. New York, USA. Freeman and Company. 937 p. doi. org/10.2307/2343822.

Stanturf J, S Mansourian, M Kleine. 2017. Implementing forest landscape restoration: A practioner's guide. Vienna, Austria. International Union of Forest Research Organization (IUFRO). 127 p.

Steel EA, MC Kennedy, PG Cunningham, JS Stanovick. 2013. Applied statistics in ecology: common pitfalls and simple solutions. Ecosphere 4(9): 1-13. doi.org/10.1890/ES1300160.1.

Toro Manríquez M. 2014. Regeneración de N. pumilio en micrositios de sectores perturbados y no perturbados por efectos del Castor canadensis Kuhl en Tierra del Fuego. Memoria para optar al Título Profesional de Ingeniero Forestal. Santiago, Chile. Facultad de Ciencias Forestales y de la Conservación de la Naturaleza, Universidad de Chile. 50 p. doi. org/10.13140/RG.2.1.1700.3042.

Toro Manríquez M, L Mestre, MV Lencinas, A Promis, G Martínez Pastur, R Soler. 2016. Flowering and seeding patterns in pure and mixed Nothofagus forests in Southern Patagonia. Ecological Processes 5(21): 1-12. doi.org/10.1186/ s13717-016-0065-1.

Valenzuela PA, E Arellano, AJ Burger, P Becerra. 2016. Using facilitation microsites as a restoration tool for conversion of degraded grasslands to Nothofagus forests in Southern Patagonia. Ecological Engineering 95(1): 580-587. doi. org/10.1016/j.ecoleng.2016.06.116.

Vidal O, M Aguayo, R Niculcar, N Bahamonde, S Radic, C San Martín, A Kusch, J Latorre, J Félez. 2015. Plantas invasoras en el Parque Nacional Torres del Paine (Magallanes, Chile): estado del arte, distribución post-fuego e implicancias en restauración ecológica. Anales del Instituto de la Patagonia 43(1): 75-96. doi.org/10.4067/S0718686X2015000100006. 